\title{
AN APPLICATION OF MOSER ITERATION TO COMPLETE MINIMAL SUBMANIFOLDS IN A SPHERE
}

\author{
LEUNG-FU CHEUNG and PUI-FAI LEUNG
}

(Received 10 April 2001; revised 14 August 2002)

Communicated by K. Wysocki

\begin{abstract}
We apply the Moser iteration method to obtain a pointwise bound on the norm of the second fundamental form from a bound on its $L^{n}$ norm for a complete minimal submanifold in a sphere. As an application we show that a complete minimal submanifold in a sphere with finite total curvature and Ricci curvature bounded away from $-\infty$ must be compact. This complements similar results of Osserman and Oliveira in the case the ambient space is the Euclidean and the hyperbolic space respectively.
\end{abstract}

2000 Mathematics subject classification: primary $53 \mathrm{C} 21$.

\section{Introduction}

In the theory of minimal surfaces in $\mathbb{R}^{3}$, an important role is played by the class of minimal surfaces with finite total Gaussian curvature. One celebrated result concerning such surfaces is

THEOREM (Osserman). Let $M^{2}$ be a minimal surface in $\mathbb{B}^{3}$ with finite total Gaussian curvature. Then $M^{2}$ is conformally equivalent to a compact Riemann surface minus a finite number of points.

This result has a generalization to the case when the ambient space is the standard hyperbolic space. In this setting, Oliveira proved

THEOREM ([8]). Let $M^{2}$ be a minimal surface in the standard hyperbolic space with $L^{2}$ second fundamental form. Then $M^{2}$ is diffeomorphic to a compact Riemann surface minus a finite number of discs.

This project was partially financed by the PolyU research grant G-T492.

(C) 2004 Australian Mathematical Society 1446-7887/04 \$A2.00+0.00 
In this note, we look at a generalization of Osserman's theorem in the case when the ambient space is positively curved. Specifically, we consider the situation where $M^{n}$ is a minimal submanifold sitting in the standard $(n+k)$-dimensional sphere with analogous curvature assumptions imposed on $M^{n}$. We are interested in understanding what topological effect such curvature assumptions will have on $M^{n}$. To be more precise, we will prove

THEOREM 1. If $M^{n}$ is a complete minimal submanifold of the sphere $S^{n+p}$ with $\int_{M}|A|^{2 n-2} d M<\infty$, then $M^{n}$ is compact.

REMARK 1. In the case when $n=2$, this condition seems optimal, since the condition becomes $\int_{M}|A|^{2} d M<\infty$.

THEOREM 2. If $M^{n}$ is a complete minimal submanifold of the sphere $S^{n+p}$ with $\int_{M}|A|^{n} d M<\infty$ and $\inf _{M} \operatorname{Ric}(M)>-\infty$, then $M^{n}$ is compact.

This result together with the results of Osserman and Oliveira forms a rather complete description of the topology of a minimal surface in a space form with $L^{2}$ second fundamental form (that is, $|A| \in L^{2}\left(M^{2}\right)$ ).

\begin{tabular}{|c|l|}
\hline Ambient Space & \multicolumn{1}{|c|}{ Result } \\
\hline hyperbolic space & $\begin{array}{l}M^{2} \text { is conformally equivalent to a compact Riemann } \\
\text { surface minus finitely many discs }\end{array}$ \\
\hline Euclidean space & $\begin{array}{l}M^{2} \text { is conformally equivalent to a compact Riemann } \\
\text { surface minus finitely many points }\end{array}$ \\
\hline sphere & $M^{2}$ is compact \\
\hline
\end{tabular}

Related to Theorem 2 is the following theorem of Shen and Wang ([11, 12]):

THEOREM. Let $M^{n}$ be a compact minimal submanifold of the unit sphere $S^{n+p}$. Suppose $\operatorname{Ric}(X, X) \geq 0$ (where $X$ is any unit tangent vector). Then there exists a constant $C(n)$ depending only on $n$ such that if $\int_{M}|A|^{n} d M<C(n)$ then $|A|$ is identically equal to zero and $M^{n}$ is totally geodesic.

We remark that Theorem 2 can be used to weaken the compactness assumption in the theorem of Shen and Wang to that of completeness.

\section{Preliminaries}

In this section, all the tools needed in the subsequent proofs will be collected. 
2.1. Simons' inequality. Consider a minimal submanifold $M^{n}$ immersed in $S^{n+p}$ and denote by $h_{i j}^{\alpha}(1 \leq i, j \leq n, 1 \leq \alpha \leq p)$ its second fundamental form. Then the following Simons' inequality holds:

$$
\Delta|A| \geq n|A|-(2-1 / p)|A|^{3} \quad \text { in distributional sense, }
$$

where $|A|$ is the length of the second fundamental form of $M^{n}$ (see Chern [3, page 41], Berard [1, page 49]).

This can be seen by using the computations of Chern on an estimate of $\sum h_{i j}^{\alpha} \Delta h_{i j}^{\alpha}$ followed by Kato's inequality (see [2] and also Berard [1]) for the second fundamental form $h_{i j}^{\alpha}$. It follows that

$$
\Delta|A|+C(p)|A|^{3} \geq 0 .
$$

2.2. Isoperimetric-type inequality. Another important ingredient of our proof is the following isoperimetric inequality of $\mathrm{Li}$ and $\mathrm{Yau}$ [7]:

LEMMA 1 ( $\mathrm{Li}$ and Yau [7]). Let $M$ be a complete noncompact Riemannian manifold without boundary of dimension $n$. Let $R(x)$ denote the pointwise lower bound of the Ricci curvature, that is, $\operatorname{Ric}(x) \geq R(x) g_{i j}$ and let $R_{-}(x)=\max \{0,-R(x)\}$ be the negative part of $R(x)$. If the geodesic ball of radius $r$ centered at $y \in M$ is denoted by $B_{r}(y)$, its volume is denoted by $V_{y}(r)$, and the area of its boundary is denoted by $A_{y}(r)$, then for any $p \geq n-1$ there exist constants $C_{1}, C_{2}>0$ depending only on $n$ such that for any $r>0$,

$$
A_{y}(r) \leq C_{1} r^{n-1}+C_{2} r^{2 n-4} V_{y}^{(p-n+1) / p}(r) \int_{0}^{r}\left(\int_{B_{t}(r)} R_{-}^{p} d M\right)^{(n-1) / p} d t .
$$

2.3. Ricci lower bound estimate for minimal submanifolds. The last result we will need is the following estimate of Leung [6]:

LEMMA 2 (Leung [6]). Let $M^{n}$ be a minimal submanifold in $S^{n+p}$ with constant sectional curvature $K$. Then the following inequality holds

$$
\operatorname{Ric}(X, X) \geq \frac{n-1}{n}\left\{n K-|A|^{2}\right\},
$$

where $X$ is any unit tangent vector.

In the next section, we will prove the main result, that is, Theorem 3 . This is done by using a technical lemma on the decay of $|A|(p)$ (to be proved in the Appendix using Moser's iteration technique on Simons' inequality while keeping control on the support of test functions) as $p$ escapes compact sets on $M$. Having done this, the Ricci lower bound estimate shows then that the Ricci curvature of $M$ is positively pinched outside some compact set of $M$. A modification of Myer's theorem yields subsequently the compactness. 


\section{Proof of main theorem}

We shall prove in this and the next section the following two theorems which complement a result of Oliveira [8].

THEOREM 3. Let $M^{n}$ be a complete noncompact minimal submanifold in $S^{n+p}$ with $\int_{M}|A|^{n} d M<\infty$ and let $\operatorname{Ric}(X, X) \geq(n-1) \kappa$ (where $X$ is any unit tangent vector and $\kappa$ a constant). Then for any point $q \in M$,

$$
\sup _{M \backslash B_{R}(q)}|A| \rightarrow 0 \text { as } R \rightarrow \infty,
$$

where $B_{R}(q)$ is a geodesic ball of radius $R$ centered at $q \in M$.

THEOREM 4. Let $M^{n}$ be a complete noncompact minimal submanifold in $S^{n+p}$ and suppose that $\int_{M}|A|^{2 n-2} d M<\infty$. Then for any point $q \in M$,

$$
\sup _{M \backslash B_{R}(q)}|A| \rightarrow 0 \text { as } R \rightarrow \infty,
$$

where $B_{R}(q)$ is a geodesic ball of radius $R$ centered at $q \in M$.

Proof OF THEOREM 3 (the case $n \geq 3$ ). Our key analytical tool is the following lemma, which will be proved in Appendix via Moser iteration:

LEMMA 3. Let $M^{n}$ be a minimal submanifold such that smooth functions supported in geodesic balls of radius $r \leq \tilde{r}$ ( $\tilde{r}$ is independent of the center $y$ ) satisfy the generalized Sobolev inequality of Hoffman and Spruck [5]. Then there exist $\epsilon>0$, $c>0, t>0$, where $t \leq \tilde{r}$, such that for each geodesic ball $B_{t}(p) \subset \subset M$, the following holds:

$$
\text { if }\left\{\int_{B_{i}(p)}|A|^{n}\right\}^{1 / n} \leq \epsilon, \text { then }|A|(p) \leq c\left\{\int_{B_{t}(p)}|A|^{n}\right\}^{1 / n} .
$$

To prove Theorem 3, note that since the Ricci curvatures of $M^{n}$ are greater than or equal to $(n-1) \kappa$, by Bishop's theorem, for all $r>0, x \in M$,

$$
V_{x}(r) \leq V_{\kappa}(r)
$$

where $V_{k}(r)$ is the volume of a ball with radius $r$ in the space form with constant sectional curvatures equal to $\kappa$.

Now, a theorem of Hoffman and Spruck [5] asserts the existence of a number $\theta>0$ depending only on the injectivity radius and the sectional curvature of the ambient 
space (and hence only on $K$ ) such that for any $x$ and $r>0$ satisfying $V_{x}(r)<\theta$, a generalized Sobolev inequality holds for $C_{0}^{\infty}$ functions compactly supported in $B_{r}(x)$. Choosing $V_{\kappa}(\tilde{r})<\theta$ implies then the existence of the radius $\tilde{r}$ with properties required by Lemma 3 .

Choosing geodesic balls of $M^{n}$ with radii $r \leq \tilde{r}$, we can apply Lemma 3. Fix $q \in M$, since $\int_{M}|A|^{n} d M<\infty$, there exists $\tilde{R}>0$ such that $R \geq \tilde{R}$ implies

$$
\int_{M \backslash B_{R}(q)}|A|^{n} d M \leq \epsilon,
$$

where $\epsilon$ is the constant in Lemma 3. Take any $B_{\imath}(p) \subset \subset M \backslash B_{R}(q)$ with $t \leq \tilde{r}$; then Lemma 3 implies $|A|(p) \leq c \epsilon$. Letting $\epsilon$ go to zero completes the proof.

ProOF OF THEOREM 4 (the case $n \geq 3$ ). Applying Lemma 2 to a complete minimal submanifold $M^{n}$ in $S^{n+p}$ we have $\operatorname{Ric}(X, X) \geq-((n-1) / n) K|A|^{2}$ (where $X$ is a unit tangent vector of $M)$ and hence we conclude that the integral $\int_{B_{y}(r)}|A|^{2 p} d M$ satisfies

$$
\int_{B_{y}(r)}|A|^{2 p} d M \geq \text { constant } \times \int_{B_{y}(r)} R_{-}^{p} d M .
$$

Next choosing $p=n-1$ in Lemma 1, we have

$$
\begin{aligned}
A_{y}(r) & \leq C_{1} r^{n-1}+C_{2} r^{2 n-4} \int_{0}^{r}\left(\int_{B_{y}(r)} R_{-}^{n-1} d M\right) d t \\
& \leq C_{1} r^{n-1}+C_{2} r^{2 n-4} \int_{0}^{r}\left(\int_{M}|A|^{2 n-2} d M\right) d t,
\end{aligned}
$$

where $C_{1}, C_{2}$ denotes constants independent of the choice of the center $y$ as in Lemma 1. From this and the hypothesis that $|A|$ is in $L^{2 n-2}(M)$, it follows that

$$
A_{y}(r) \leq C_{1} r^{n-1}+C_{3} r^{2 n-3} \text {. }
$$

Recall that for almost all $r>0$,

$$
\frac{d V_{y}(r)}{d r}=A_{y}(r)
$$

hence integrating the above inequality (2) gives the estimate

$$
V_{y}(r) \leq \frac{C_{1}}{n} r^{n}+\frac{C_{3}}{2 n-2} r^{2 n-2}
$$

which implies $V(r) \leq C r^{n}$ for $r \leq 1$, where $C:=C_{1} / n+C_{3} /(2 n-2)$. We have omitted the subscript $y$ since the estimate is independent of the choice of the center $y$. 
The last inequality and the monotonicity of the volume of geodesic balls together imply that smooth functions supported in subsets of geodesic balls $B_{r}(y)$ satisfy the hypothesis for the Sobolev inequality, provided $r \leq r_{0}$ for some $r_{0}>0$. Having established this fact, the rest of the proof is completely analogous to that of Theorem 3 except that for the initial step of Moser iteration one starts with the assumption (see for example (17) in Appendix) $\int_{M}|A|^{2 n-2} d M<\infty$ instead of $\int_{M}|A|^{n} d M<\infty$. However, from the fact that $\int_{M}|A|^{2 n-2} d M<\infty$ and $V(r) \leq C r^{n}$, one can conclude

$$
\int_{M \backslash B_{R}(q)}|A|^{n} d M \rightarrow 0 \text { as } R \rightarrow \infty
$$

(where $q$ is an arbitrary but fixed point in $M$ ), which is all that is needed to move terms involving higher $L^{p}$ norms in inequality (17) of Appendix and other related inequalities to the relevant side in the Moser iteration. The fact (5) follows from our assumption because for $r \leq 1$, we have $V(r) \leq C r^{n} \leq C$, hence

$$
\int_{B_{r}}|A|^{n} d M \leq\left(\int_{B_{r}}|A|^{2 n-2} d M\right)^{n /(2 n-2)} V(r)^{1-(n /(2 n-2))} \leq C r^{(1-(n /(2 n-2 t)) ! n}<\infty .
$$

As a conclusion, for $B_{r}$ sitting outside large compact set $\overline{B_{R}(q)}$, one has

$$
\int_{B_{r}}|A|^{2 n-2} d M \rightarrow 0
$$

which implies $\int_{B_{r}}|A|^{n} d M \rightarrow 0$. Having established this, the rest follows the proof of Theorem 3.

\section{The case $n=2$}

Decay estimate under $\|\boldsymbol{A}\|_{2}$ bound. In the case when the dimension of the minimal submanifold is two, Theorem 3 covers Theorem 4 . It therefore suffices to prove Theorem 4 . To this end, we need the following stability result for minimal surfaces

PROPOSITION 1. If $M^{2} \rightarrow S^{2+p}$ is a minimal surface satisfying the condition $\int_{M}|A|^{2} d M<\infty$, then it is stable over small balls sitting outside some large compact set $\bar{B}_{T}\left(p_{0}\right)$ ( $p_{0}$ is some arbitrary but fixed point on the surface) for which $\int_{M \backslash B_{T}\left(p_{0}\right)}|A|^{2} d M$ is sufficiently small. (More precisely, there exist $T, \tilde{r}>0$ depending only on $\int_{M}|A|^{2} d M$ and $M$ but not on the choice of the center of the geodesic balls $B_{r}(p)$ such that for all $r$, if $0<r \leq \tilde{r}$ and $B_{r}(p) \subset \subset M \backslash B_{T}\left(p_{0}\right)$, then $B_{r / 2}(p)$ is a stable minimal surface.) 
PROOF. Without loss of generality we assume that the sectional curvatures $K$ of $S^{n+p}$ satisfy $K \equiv 1$. By the isoperimetric inequality of $\mathrm{Li}$ and Yau (see inequality (4) in Section 3), we have quadratic growth for geodesic balls, that is, $V_{p}(r) \leq \widetilde{C} r^{2}$ (where $\widetilde{C}$ depends on $\int_{M}|A|^{2} d M$ and is independent of the choice of the point $p$.)

We follow Oliveira [8] (the first inequality on page 14 of his paper) and apply the $L^{1}$ Sobolev inequality (that is, (3.3) of Oliveira [8]) to certain class of test functions $\xi$ (to be specified later) satisfying the condition $\operatorname{supp} \xi \subset \subset B_{r}(p)$, where $r \leq \tilde{r}$ with $\tilde{r}$ defined as in the proof of Theorem 4 to get

$$
\left\|\xi|A|^{2}\right\|_{2} \leq 2 \widetilde{C}_{1}\left(\|\xi|A| \nabla|A|\|_{1}+\left\||A|^{2} \nabla \xi\right\|_{1}\right), \quad \forall \xi \in C_{0}^{\infty}\left(B_{r}(p)\right) .
$$

Combining with the Cauchy-Schwarz inequality on the right-hand-side, this becomes

$$
\left\|\xi|A|^{2}\right\|_{2, B_{r}(p)} \leq 2 \widetilde{C}_{1}\|\chi|A|\|_{2, B_{r}(p)}\left(\|\xi \nabla|A|\|_{2, B_{r}(p)}+\||A| \nabla \xi\|_{2, B_{r}(p)}\right),
$$

where $\chi=$ characteristic function of $\operatorname{supp} \xi$.

On the other hand, Simons' inequality multiplied by $\xi^{2}|A|^{q}$ (where $q=1$ ) and integrated, yields (see $[8,(3.14)]$ ),

$$
\|\xi \nabla|A|\|_{2, B_{r}(p)} \leq \widetilde{C}_{2}\left(\left\|\xi|A|^{2}\right\|_{2, B_{r}(p)}+\||A| \nabla \xi\|_{2, B_{r}(p)}\right) .
$$

Combining the above inequalities, we have

$$
\begin{aligned}
\|\xi \nabla|A|\|_{2, B_{r}(p)} \leq & 2 \widetilde{C}_{2} \widetilde{C}_{1}\|\chi|A|\|_{2, B_{r}(p)}\left(\|\xi \nabla|A|\|_{2, B_{r}(p)}+\||A| \nabla \xi\|_{2, B_{r}(p)}\right) \\
& +\widetilde{C}_{2}\||A| \nabla \xi\|_{2, B_{r}(p) .}
\end{aligned}
$$

By taking $B_{r}(p) \subset M \backslash B_{T}\left(p_{0}\right)$ for some large number $T$, we obtain $\|\chi \mid A\|_{2, B_{r}(p)} \leq$ $1 /\left(4 \widetilde{C}_{1} \widetilde{C}_{2}\right)$ which implies

$$
\|\xi \nabla|A|\|_{2, B_{r}(p)} \leq \widetilde{C}_{3}\left\|\left|A\|\nabla \xi \mid\|_{2, B_{r}(p)}\right.\right.
$$

(see $[8,(3.18)])$.

To prove stability, choose a non-negative function $\xi$ such that

$$
\xi(x)= \begin{cases}1 & x \in B_{3 r / 4}(p) \\ 0 & x \in M \backslash B_{r}(p)\end{cases}
$$

with $|\nabla \xi| \leq 8 / r$ and consider arbitrary $\varphi \in C_{0}^{\infty}\left(B_{r / 2}(p)\right)$. By the $L^{1}$ Sobolev 
inequality,

$$
\begin{aligned}
\||A| \varphi \xi\|_{2, B_{r}(p)} \leq & C_{s}\|\nabla(|A||\varphi| \xi)\|_{1, B_{r}(p)} \quad \text { (where } C_{s} \text { is the Sobolev constant) } \\
\leq & C_{s}\left(\int_{B_{r}(p)}|\varphi||\nabla(|A| \xi)|+\int_{B_{r}(p)} \xi|A||\nabla \varphi|\right) \\
\leq & C_{s}\left(\int_{B_{r}(p)}|\varphi| \xi|\nabla| A||+\int_{B_{r}(p)}|\varphi\|A\| \nabla \xi|+\int_{B_{r}(p)}|A| \xi|\nabla \varphi|\right) \\
\leq & C_{s}\left\{\|\xi|\nabla| A\|\left\|_{2, B_{r}(p)}\right\| \varphi\left\|_{2, B_{r}(p)}+\right\| \varphi\left\|_{2, B_{r}(p)}\right\||A| \nabla \xi \|_{2, B_{r}(p)}\right. \\
& \left.+\||A| \xi\|_{2, B_{r}(p)}\|\nabla \varphi\|_{2, B_{r}(p)}\right\} .
\end{aligned}
$$

Using the fact that $\xi \equiv 1$ on $B_{3 r / 4}(p)$,

$$
\begin{aligned}
\||A| \varphi\|_{2, B_{r / 2}(p)} \leq & \||A| \varphi \xi\|_{2, B_{3 / 4}(p)} \\
\leq & C_{s}\left\{\|\xi|\nabla| A\|\left\|_{2, B_{r}(p)}\right\| \varphi\left\|_{2, B_{r}(p)}+\right\| \varphi\left\|_{2, B_{r}(p)}\right\||A| \nabla \xi \|_{2, B_{r}(p)}\right. \\
& \left.+\||A| \xi\|_{2, B_{r}(p)}\|\nabla \varphi\|_{2, B_{r}(p)}\right\} .
\end{aligned}
$$

Now we study the terms on the right-hand side of this inequality. First we observe that

1. $\||A| \xi\|_{2, B_{r}(p)}\|\nabla \varphi\|_{2, B_{r}(p)} \leq\left(1 /\left(6 C_{s}\right)\right)\|\nabla \varphi\|_{2, B_{r}(p)}$, if we choose

$$
\|A\|_{2, B,(p)} \leq 1 /\left(6 C_{s}\right) \text {. }
$$

2. From (7), $\left\|\xi|\nabla| A\left|\|\|_{2, B_{r}(p)} \leq \widetilde{C}_{3}\||A| \nabla \xi\|_{2, B_{r}(p)} \leq 1 /\left(6 C_{s}\right)\right.\right.$ by choosing

$$
\|A\|_{2, B_{r}(p)} \leq r /\left(48 C_{s} \widetilde{C}_{3}\right) \text {. }
$$

3. $\||A| \nabla \xi\|_{2} \leq\|A\|_{2, B_{r}(p)} 8 / r \leq 1 /\left(6 C_{s}\right)$ by choosing $\|A\|_{2, B_{r}(p)} \leq r /\left(48 C_{s}\right)$.

Putting all these inequalities back into ( 8 ), we have

$$
\begin{aligned}
\||A| \varphi\|_{2, B_{r / 2}(p)} & \leq\left\{2\|\varphi\|_{2, B_{r}(p)}+\|\nabla \varphi\|_{2, B_{r}(p)}\right\} / 6 \\
& =\left\{2\|\varphi\|_{2, B_{r / 2}(p)}+\|\nabla \varphi\|_{2, B_{r / 2}(p)}\right\} / 6 .
\end{aligned}
$$

Next, apply the isoperimetric inequality of Li and Yau to functions

$$
\varphi \in C_{0}^{\infty}\left(B_{r / 2}(p)\right) \quad \text { (with } r \leq \tilde{r} \text { ) }
$$

together with the Sobolev inequality of Hoffman and Spruck and get the following estimate on $\|\varphi\|_{2, B_{r / 2}(p)}$ :

$$
\begin{aligned}
\|\varphi\|_{2, B_{r / 2}(p)} & \leq C_{s}\|\nabla \varphi\|_{1, B_{r / 2}(p)} \leq C_{s} \int_{B_{r / 2}(p)}|\nabla \varphi| d M \\
& \leq C_{s}\|\nabla \varphi\|_{2, B_{r / 2}(p)} V_{p}(r / 2)^{1 / 2} \\
& \leq C_{s}\|\nabla \varphi\|_{2, B_{r / 2}(p)} \widetilde{C}^{1 / 2} r / 2=C_{6} r\|\nabla \varphi\|_{2, B_{r / 2}(p)} .
\end{aligned}
$$


This estimate, when applied to (9), yields

$$
\||A| \varphi\|_{2, B_{r / 2}(p)} \leq(1 / 6)\left\{2 C_{6} r\|\nabla \varphi\|_{2, B_{r / 2}(p)}+\|\nabla \varphi\|_{2, B_{r / 2}(p)}\right\} .
$$

Finally, choosing $\tilde{r} \leq 1 /\left(4 C_{6}\right)$, we obtain

$$
\begin{aligned}
\|A \mid \varphi\|_{2, B_{r / 2}(p)} & \leq(1 / 6)\left\{(1 / 2)\|\nabla \varphi\|_{2, B_{r / 2}(p)}+\|\nabla \varphi\|_{2, B_{r / 2}(p)}\right\} \\
& =(1 / 4)\left\{\|\nabla \varphi\|_{2, B_{r / 2}(p)} \leq(1 / 3)\|\nabla \varphi\|_{2, B_{r / 2}(p)}\right.
\end{aligned}
$$

which implies

$$
\int_{B_{r / 2}(p)}|A|^{2} \varphi^{2} d M \leq \frac{1}{9} \int_{B_{r / 2}(p)}|\nabla \varphi|^{2} d M
$$

this completes one half of the stability inequality. The other half follows by applying (10) again to $\varphi$ :

$$
\|\varphi\|_{2} \leq C_{6} \tilde{r}\|\nabla \varphi\|_{2} \leq\|\nabla \varphi\|_{2} / 2
$$

this upon squaring gives

$$
\int_{M} 2 \varphi^{2} \leq \int_{M} \frac{1}{2}|\nabla \varphi|^{2}
$$

Adding (12) to (13) gives the stability inequality in the ball $B_{r / 2}$.

Having obtained the stability of the surface restricted to balls of radius $r / 2$ sitting inside $M \backslash B_{T}\left(p_{0}\right)$, we can follow Oliveira [8] to use Schoen's result [10, Theorem 3] and obtain the existence of constants $C_{7}$ and $\mu$ not depending on $p$ with the following property:

$$
0<\mu \leq 1 / 2 \text { and } \sup _{B_{\mu r}}|A| \leq C_{7}
$$

Furthermore, the Li-Yau isoperimetric inequality provides a bound on the volume of balls $B_{\mu r}(p)$, that is,

$$
V_{p}(\mu r) \leq \widetilde{C} \mu^{2} r^{2} \leq \widetilde{C}_{7}
$$

because $\mu r \leq 1$ by definition.

Inequalities (14) and (15) enable us to follow Oliveira (see also Appendix) to obtain the decay in the dimension 2 case. This finishes the proof of Theorem 4 in the dimension 2 case (see Oliveira [8, pages 15-16]). 


\section{Proof of the two main theorems}

Theorem 3 and Theorem 4 when combined with the following lemma will give our main result.

LEMMA 4. If $M^{n}$ is a complete Riemannian manifold with

$$
\operatorname{Ric}(X, X) \geq(n-1) \kappa>0 \quad \text { (where } X \text { is any unit tangent vector) }
$$

outside some compact subset $G$ of $M^{n}$, then $M^{n}$ is compact.

Proof. Let $B_{T}(p)$ be a geodesic ball with radius $T$ centered at a point $p$ in the interior of $G$ and containing $G$. By definition, the boundary of this ball is a connected set. Take $\tilde{p} \in$ the closure of $B_{T}(p)$, then we have $d(p, \tilde{p}) \leq T$. If $\tilde{p} \in M \backslash B_{T}(p)$, then the completeness of $M$ guarantees the existence of an arc-length parametrized geodesic $\gamma$ joining $\tilde{p}$ to $q \in \partial B_{T}(p)$ realizing the distance $d\left(\tilde{p}, \partial B_{T}(p)\right)$. This geodesic lies outside $B_{T}(p)$ from the definition of $B_{T}(p)$. But the proof of Myer's Theorem implies $L(\gamma) \leq \pi / \sqrt{\kappa}+T$, since $\operatorname{Ric}\left(\gamma^{\prime}, \gamma^{\prime}\right) \geq(n-1) \kappa$ outside $G$. Hence by the triangle inequality, we have $d(\tilde{p}, p) \leq d(\tilde{p}, q)+d(q, p) \leq \pi / \sqrt{\kappa}$. It follows that $\operatorname{diam}(M)<2 \pi / \sqrt{\kappa}+2 T$.

Now the two main theorems (Theorems 1 and 2) can be proved by contradiction. Suppose $M$ is non-compact, fix a point $p_{0} \in M^{n}$ and denote the geodesic distance from this point by $R$. By Theorems 3 and $4,|A| \rightarrow 0$ as $R \rightarrow \infty$, which implies that outside a big compact subset $G$ of $M^{n},|A|^{2} \leq n K / 2$. Hence by Lemma 2 the Ricci curvatures of $M^{n}$ is bounded away from zero outside a big compact subset of $M^{n}$. A contradiction by Lemma 4 .

\section{Appendix (proof of Lemma 3)}

The proof of Lemma 3 follows the standard Moser iteration procedure. However, care has to be taken to guarantee that the volumes of the geodesic balls can be bounded uniformly from above by $\mathrm{Cr}^{n}$.

PROOF ( $n \geq 3)$. In the sequel, we shall occasionally denote $|A|$ by $u$.

Choose a geodesic ball with radius $r$ and centered at $p$, by the hypothesis of Lemma 3 all functions on $M^{n}$ supported in $B_{r}(p)$ with $r \leq \tilde{r}$ ( $p$ is arbitrary here!) satisfy the generalized Sobolev inequality of Hoffman and Spruck.

Restricting to functions in $C_{0}^{\infty}\left(B_{r}(p)\right)$, we can apply the standard Moser iteration argument to the Simons' inequality to obtain a decay estimate of, $|A|(p)$ (see for example [8]). We shall now give the details. 
Let $s>2$ and $\xi \in C_{0}^{\infty}\left(B_{r}(p)\right), \chi$ denote the characteristic function of supp $\xi$. Using the Hölder inequality, we can estimate $\int_{M} \xi^{2}|A|^{2} u^{2 q} d M$ and obtain (for simplicity $d M$ will be omitted in the sequel)

$$
\begin{aligned}
\int_{M} \xi^{2}|A|^{2} u^{2 q} & \leq\left\{\int_{M}\left(\chi|A|^{2}\right)^{s / 2}\right\}^{2 / s}\left\{\int_{M}\left(\xi^{2} u^{2 q}\right)^{s /(s-2)}\right\}^{(s-2) / 2} \\
& =\left\|\chi|A|^{2}\right\|_{s / 2}\left\|\xi u^{q}\right\|_{2 s /(s-2)}^{2} .
\end{aligned}
$$

On the other hand, Simons' inequality yields the following inequality after multiplication through by $u^{2 g-1} \xi^{2}$ and estimating in a standard way (see [8]):

$$
\left\|\nabla\left(\xi u^{q}\right)\right\|_{2} \leq C_{8} \sqrt{q}\left\{\left\|u^{q}|\nabla \xi|\right\|_{2}+\left\|u^{q+1} \xi\right\|_{2}\right\} .
$$

Applying the Sobolev inequality of Hoffman and Spruck [5], that is,

$$
\|f\|_{n m /(n-m)} \leq \widetilde{C}_{s} \frac{m(n-1)}{n-m}\|\nabla f\|_{m}, \quad \forall f \in C_{0}^{\infty}\left(B_{r}(p)\right), \quad \forall m<n,
$$

with $m=2$ and $\xi u^{q}$ replacing $f$ gives

$$
\begin{aligned}
\left\|\xi u^{q}\right\|_{2 n / n-2} & \leq C_{s}\left\|\nabla\left(\xi u^{q}\right)\right\|_{2} \quad\left(\text { where } \quad C_{s}=\widetilde{C}_{s} \frac{2(n-1)}{n-2}\right) \\
& \leq C_{s} C_{8} \sqrt{q}\left\{\left\|u^{q} \mid \nabla \xi\right\|_{2}+\left\|u^{q+1} \xi\right\|_{2}\right\} \\
& \leq \widehat{C} \sqrt{q}\left(\left\|u^{q}|\nabla \xi|\right\|_{2}+\left\|\chi|A|^{2}\right\|_{s / 2}^{1 / 2}\left\|\xi u^{q}\right\|_{2 s /(s-2)}\right)
\end{aligned}
$$

with $\widehat{C}=C_{s} C_{8}$ which depends on $n$.

Now take $s=n$ and $q=n / 2$. Then the above inequality reads

$$
\left\|\xi u^{n / 2}\right\|_{2 n /(n-2)} \leq \widehat{C}_{1} \sqrt{n}\left\{\left\|u^{n / 2}|\nabla \xi|\right\|_{2}+\left\|\chi|A|^{2}\right\|_{n / 2}^{1 / 2}\left\|\xi u^{n / 2}\right\|_{2 n /(n-2)}\right\},
$$

where $\widehat{C}_{1}=\widehat{C} / \sqrt{2}$.

Next, we try to absorb the last term of this expression to the other side of the inequality by requiring (this choice determines the $\epsilon$ to be fixed later)

$$
\int_{B_{r}(p)}\left(|A|^{n} d M\right)^{1 / n} \leq \frac{1}{2 \widehat{C}_{1} \sqrt{n}}
$$

Hence we obtain

$$
\left\|\xi u^{n / 2}\right\|_{2 n /(n-2)} \leq C_{*}\left\|u^{n / 2}|\nabla \xi|\right\|_{2} \leq C_{*} \sup _{B_{r}(p)}|\nabla \xi|\left\{\int_{B_{r}(p)}|A|^{n}\right\}^{1 / 2},
$$


where $C_{*}=2 \widehat{C}_{1} \sqrt{n}$. Choose $\xi$ such that $\xi \equiv 1$ on $B_{3 r / 4}(p)$ and $\xi \equiv 0$ outside $B_{r}(p)$, $|\nabla \xi| \leq 8 / r$. Then we have

$$
\left\||A|^{n / 2}\right\|_{2 n /(n-2), B_{3 r / 4}(p)} \leq \frac{8 C_{*}}{r}\left\{\int_{B_{r}(p)}|A|^{n}\right\}^{1 / 2} \leq 8 c_{*}\left\{\int_{B_{r}(p)}|A|^{n}\right\}^{1 / 2},
$$

where $c_{*}=C_{*} / r=\left(2 \widehat{C}_{1} / r\right) \sqrt{n}$. Choose $\epsilon \geq 0$ such that $\epsilon<\left(1 / 8 c_{*}\right)^{2 / n}$ and $\epsilon<1 /\left(2 \widehat{C}_{1} \sqrt{n}\right)$, hence if $\int_{B,(p)}|A|^{n} \leq \epsilon^{n}$, then the following inequalities hold:

$$
8 c_{*}\left\{\int_{B_{r}(p)}|A|^{n}\right\}^{1 / 2} \leq 1 \text { and } \widehat{C}_{1} \sqrt{n}\left\{\int_{B_{r}(p)}|A|^{n}\right\}^{1 / n}<\frac{1}{2} .
$$

With this choice of $\epsilon$, inequality (18) gives for all $\xi \in C_{0}^{\infty}\left(B_{3 r / 4}(p)\right), \chi:=\operatorname{supp} \xi$,

$$
\left\{\int_{B_{3 r / 4}(p)}|A|^{n^{2} /(n-2)}\right\}^{(n-2) / 2 n} \leq 1 .
$$

Because of this inequality and since for $s=n^{2} / n-2$,

$$
\left\|u^{2}\right\|_{s / 2, B_{3 / 4}(p)}^{1 / 2}=\left\|u^{n / 2}\right\|_{2 n /(n-2), B_{3 / 4}(p)}^{2 / n},
$$

inequality (16) and $s=n^{2} /(n-2)$ gives

(19) $\quad\left\|\xi u^{q}\right\|_{2 n /(n-2)} \leq \widehat{C} \sqrt{q}\left\{\left\|u^{q}|\nabla \xi|\right\|_{2}+\left\|\xi u^{q}\right\|_{2 s /(s-2)}\right\}, \quad \forall \xi \in C_{0}^{\infty}\left(B_{3 r / 4}(p)\right)$.

The next step is an interpolation argument. Recall the interpolation inequality for $L^{p}$-spaces:

$$
\left\|\xi u^{q}\right\|_{2 r / s-2} \leq \delta\left\|\xi u^{q}\right\|_{2 n / n-2}+\delta^{-\sigma}\left\|\xi u^{q}\right\|_{2}, \quad \forall \delta>0 \text { and } s \geq n .
$$

Put $s=n^{2} / n-2, \sigma=n / n-2$ in (20) and choose $\delta$ such that $\widehat{C} \delta \sqrt{q}=1 / 4$, where $\widehat{C}$ is the constant in (19). Then

$$
\delta=(4 \widehat{C} \sqrt{q})^{-1}, \quad \delta^{-\sigma}=(4 \widehat{C} \sqrt{q})^{\sigma} .
$$

From (19) and (20), we have for every $\xi \in C_{0}^{\infty}\left(B_{3 r / 4}(p)\right)$

$$
\begin{aligned}
\left\|\xi u^{q}\right\|_{2 n / n-2} & \leq \widehat{C} \sqrt{q}\left\{\left\|u^{q}|\nabla \xi|\right\|_{2}+\frac{1}{4 \widehat{C} \sqrt{q}}\left\|\xi u^{q}\right\|_{2 n / n-2}+(4 \widehat{C} \sqrt{q})^{\sigma}\left\|\xi u^{q}\right\|_{2}\right\} \\
& \leq \tilde{c} \sqrt{q}\left\{\left\|u^{q}|\nabla \xi|\right\|_{2}+q^{\sigma / 2}\left\|\xi u^{q}\right\|_{2}\right\},
\end{aligned}
$$

where $c:=(4 \widehat{C})^{1+\sigma} / 3$. 
To proceed further, let $\xi_{i} \in C_{0}^{\infty}\left(B_{i}\right)$, where $B_{i}:=B_{\left(1 / 4+1 / 2^{i+1}\right) r}(p), \xi_{i} \equiv 1$ on $B_{i+1}, \xi_{i} \equiv 0$ on $M \backslash B_{i}, \chi_{i}:=$ characteristic function of supp $\xi_{i}$ and such that $\left|\nabla \xi_{i}\right| \leq$ $2 \cdot 2^{i+2} / r$, then the previous inequality gives

$$
\begin{aligned}
\left\|\xi_{i} u^{q}\right\|_{2 \sigma} & \leq \tilde{c} \sqrt{q}\left\{\frac{2}{r} 2^{i+2}\left\|\chi_{i} u^{q}\right\|_{2}+q^{\sigma / 2}\left\|\chi_{i} u^{q}\right\|_{2}\right\} \\
& \leq 2 \tilde{c} \sqrt{q}\left\{\frac{2^{i+2}}{r}\left\|\chi_{i} u^{q}\right\|_{2}+q^{\sigma / 2}\left\|\chi_{i} u^{q}\right\|_{2}\right\} \\
& =2 \tilde{c} \sqrt{q}\left\|\chi_{i} u^{q}\right\|_{2}\left(\frac{2^{i+2}}{r}+q^{\sigma / 2}\right) \\
& =\frac{2 \tilde{c} \sqrt{q}}{r}\left\|\chi_{i} u^{q}\right\|_{2}\left(2^{i+2}+r q^{\sigma / 2}\right) \\
& \leq \frac{2 \tilde{c} \sqrt{q}}{r}\left\|\chi_{i} u^{q}\right\|_{2}\left(2^{i+2}+q^{\sigma / 2}\right)
\end{aligned}
$$

as we can assume without loss of generality that $r \leq 1$. Since $\operatorname{supp} \xi_{i} \subset\{x \in M$ : $\left.\xi_{i-1}(x) \equiv 1\right\},(21)$ implies

$$
\left\{\int_{B_{i+1}} u^{2 q \sigma}\right\}^{1 / 2 \sigma} \leq \frac{2 \tilde{c} \sqrt{q}}{r}\left\{\int_{B_{i}} u^{2 q q}\right\}^{1 / 2}\left\{2^{i+2}+q^{\sigma / 2}\right\}
$$

squaring this implies

$$
\begin{aligned}
\left\{\int_{B_{i+1}} u^{2 q \sigma}\right\}^{1 / \sigma} & \leq\left\{\frac{2 \tilde{c} \sqrt{q}}{r}\right\}^{2}\left\{\int_{B_{i}} u^{2 q}\right\}\left\{2^{i+2}+q^{\sigma / 2}\right\}^{2} \\
& =c^{\prime} q\left(\int_{B_{i}} u^{2 q}\right)\left(2^{i+2}+q^{\sigma / 2}\right)^{2},
\end{aligned}
$$

where $c^{\prime}=(2 \widetilde{c} / r)^{2}$ and $c^{\prime}$ depends only on $r$ and $n$.

Next, let $2 q=n \sigma^{i}$. Then

$$
\begin{aligned}
c^{\prime} q\left(2^{i+2}+q^{\sigma / 2}\right)^{2} & =\frac{c^{\prime} n \sigma^{i}}{2}\left\{2^{i+2}+\left(n \sigma^{i} / 2\right)^{\sigma / 2}\right\}^{2} \\
& =\frac{c^{\prime} n \sigma^{i}}{2}\left\{2^{i+2}+(n / 2)^{\sigma / 2} \sigma^{i \sigma / 2}\right\}^{2} \leq c_{*}^{i},
\end{aligned}
$$

where $c_{*}^{i}$ depends only on $n$ and $r$.

To conclude our iteration process, we have

$$
\begin{gathered}
\left\{\int_{B_{i+1}}|A|^{n \sigma^{i+1}}\right\}^{1 / \sigma} \leq c_{*}^{i} \int_{B_{i}}|A|^{n \sigma^{i}} \\
\left\{\int_{B_{i+1}}|A|^{n \sigma^{i+1}}\right\}^{1 / \sigma^{i+1}} \leq c_{*}^{i / \sigma^{i}}\left\{\int_{B_{i}}|A|^{n \sigma^{i}}\right\}^{1 / \sigma^{i}} .
\end{gathered}
$$


Denoting the left-hand side of the last inequality by $I_{i+1}$ and the right-hand side by $I_{i}$, we get

$$
I_{i+1} \leq c_{*}^{i / \sigma^{i}} I_{i} \leq c_{*}^{i / \sigma+(i-1) / \sigma^{i-1}} I_{i-1} \leq c_{*}^{i / \sigma^{i}+(i-1) / \sigma^{i-1}+\cdots+1 / \sigma+0} I_{0}
$$

which when passing to the limit, yields $I_{\infty} \leq c_{* *} I_{0}$, concluding the proof of Lemma 3 .

We conclude by a remark for the iteration when $n=2$.

REMARK 2 (the case $n=2$ ). In this case, one performs precisely the same iteration, but using the $L^{1}$-Sobolev inequality, that is, $\|f\|_{n /(n-1)} \leq C_{s}\|\nabla f\|_{1}$ instead of the $L^{2}$ Sobolev inequality, that is, $\|f\|_{2 n /(n-2)} \leq C_{s}\|\nabla f\|_{2}$ with some simple modifications. This leads to iteration inequality of the form

$$
\left\|\xi^{2} u^{2 q}\right\|_{n /(n-1)} \leq \text { const. } \sqrt{q}\left\{\left\|u^{q} \mid \nabla \xi\right\|_{2}+\left\|\xi u^{2}\right\|_{s / 2}^{1 / 2}\left\|\xi u^{q}\right\|_{2 s /(s-2)}\right\}
$$

with $s>2$. This last condition on $s$ requires for the initial step a higher norm bound on $|A|$ over small balls than the $L^{2}$ norm, which is provided by the stability result of Schoen [10].

\section{Acknowlegement}

The referee has informed us that P. Berard and W. Santos have proved stronger versions of the above theorems by using different methods in their paper "Curvature estimates and stability properties of CMC-submanifolds in space forms" in Matematica Contemporanea, 17 (1999), pages 77-97. More precisely, they have proved that for Theorem 1 the conclusion holds under the assumption that $\int_{M}|A|^{q} d M<\infty$ for any $q \geq n$ and for Theorem 2 the conclusion holds without the assumption on the Ricci curvature. We are very grateful to the referee who suggested that our methods are sufficiently different and interesting in themselves and may be useful in other general situations.

\section{References}

[1] P. Berard, 'Remarques sur l'équations de J. Simons' (French), in: Differential geometry. A symposium in honor of Manfredo do Carmo (eds. B. Lawson and K. Tennenblatt), Pitman Monogr. Surveys Pure Appl. Math. 52 (Longman Sci. Tech., Harlow, 1991) pp. 47-57.

[2] J. P. Bourguignon, 'The "magic" of Weitzenböck formulas', in: Variational methods (Paris, 1988) (eds. H. Berestycki, J. M. Coron and I. Ekeland) (Birkhäuser, Boston, 1990) pp. 251-271. 
[3] S. S. Chern, Minimal submanifolds in a Riemannian manifold, Lecture Notes (University of Kansas, 1968).

[4] S. S. Chern and R. Osserman, 'Complete minimal surfaces in $E^{n '}$, J. d'Analyse Math. 19 (1967), 15-34.

[5] D. Hoffman and J. Spruck, 'Sobolev and isoperimetric inequalities for Riemannian manifolds', Comm. Pure Appl. Math. 27 (1975), 715-725.

[6] P. F. Leung, 'Minimal submanifolds in a sphere', Math. Z. 183 (1983), 75-86.

[7] P. Li and S. T. Yau, 'Curvature and holomorphic mappings of complete Kähler manifolds', Comp. Math. 73 (1990), 125-144.

[8] G. Oliveira, 'Compactification of minimal submanifold of hyperbolic space', Comm. Anal. Geom. 1 (1993), 1-23.

[9] R. Osserman, 'On complete minimal surfaces', Arch. Rat. Mech. Anal. 13 (1963), 392-404.

[10] R. Schoen, 'Estimates for minimal surfaces in three dimensional manifolds', in: Seminars on minimal submanifolds (ed. E. Bombieri), Ann. of Math. Studies 103 (Princeton Univ. Press, Princeton, 1983) pp. 111-126.

[11] C. L. Shen, 'A global pinching theorem for minimal hypersurfaces in a sphere', Proc. Amer. Math. Soc. 105 (1989), 192-198.

[12] H. Wang, 'Some global pinching theorems for minimal submanifolds of the sphere', Acta Math. Sinica 31 (1988), 503-507 (in Chinese).

Department of Applied Mathematics

The Hong Kong Polytechnic University

Hung Hom Kowloon

Hongkong

e-mail: malfcheu@hkpu07.polyu.edu.hk
Department of Mathematics National University of Singapore

Lower Kent Ridge Road

Singapore 119260

Singapore

e-mail: matfredl@nus.edu.sg 
J. Aust. Math. Soc. 76 (2004) 